\title{
EFFICIENCY OF A POSTERIORI BEM-ERROR ESTIMATES FOR FIRST-KIND INTEGRAL EQUATIONS ON QUASI-UNIFORM MESHES
}

\author{
CARSTEN CARSTENSEN
}

\begin{abstract}
In the numerical treatment of integral equations of the first kind using boundary element methods (BEM), the author and E. P. Stephan have derived a posteriori error estimates as tools for both reliable computation and self-adaptive mesh refinement. So far, efficiency of those a posteriori error estimates has been indicated by numerical examples in model situations only. This work affirms efficiency by proving the reverse inequality. Based on best approximation, on inverse inequalities and on stability of the discretization, and complementary to our previous work, an abstract approach yields a converse estimate. This estimate proves efficiency of an a posteriori error estimate in the BEM on quasi-uniform meshes for Symm's integral equation, for a hypersingular equation, and for a transmission problem.
\end{abstract}

\section{INTRODUCTION}

In recent decades adaptive mesh refining proved to be a tool of high practical importance in numerical analysis of partial differential equations. The questions of how and where to perform the refinement and whether this is "efficient" (a concept to be defined) is subject of many papers, and we refer, e.g., to [1, 14, 15, 16, 17, 18, $23,24,28,29,30]$ and the references quoted therein. The framework of adaptive methods, introduced by Eriksson and Johnson $[14,15]$ for finite elements, is studied in $[3,4,5,6,7,8]$ for boundary element methods (BEM) and covers weakly singular and hypersingular integral equations, integral equations for transmission problems, and the coupling of finite elements and boundary elements. However, the questions of efficiency of the adaptive algorithms and the sharpness of the a posteriori error estimates have been studied by numerical experiments only.

In this paper we focus our attention mainly on quasi-uniform meshes (for reasons which become clear later and are discussed in §8.4) and then prove that one of the a posteriori estimates is sharp, i.e., a complementary inequality holds. We first describe a typical example and state the results obtained; the subsequent sections contain proofs, more abstract results and further applications.

Consider Symm's integral equation: Given $f$ find $\phi$ with

$$
V \phi(x)=f(x) \quad(x \in \Gamma) .
$$

Received by the editor March 22, 1994 and, in revised form, September 13, 1994. 1991 Mathematics Subject Classification. Primary 65N38, 65N15, 65R20, 45L10.

Key words and phrases. Boundary element method, a posteriori error estimate. 
Here, $\Gamma=\partial \Omega$ is a polygon, the boundary of a bounded two-dimensional Lipschitz domain $\Omega$, and

$$
V \phi(x):=-\frac{1}{\pi} \int_{\Gamma} \phi(y) \log |x-y| d s_{y}
$$

is the weakly singular single-layer potential operator.

Equation (1.1) is equivalently related to the Dirichlet problem for the Laplacian

$$
\Delta u=0 \quad \text { in } \Omega, \quad u=u_{0} \quad \text { on } \Gamma,
$$

with given Dirichlet data $u_{0}$. Indeed, using the right-hand side

$$
f(x)=u_{0}(x)-\frac{1}{\pi} \int_{\Gamma} u_{0}(y) \frac{\partial}{\partial n_{y}} \log |x-y| d s_{y} \quad(x \in \Gamma)
$$

in (1.1), one obtains $\phi=\frac{\partial u}{\partial n}$, i.e., the unknown $\phi$ in (1.1) is the normal derivative of the solution $u$ of the Dirichlet problem. Moreover, once $\left.u\right|_{\Gamma}$ and $\phi$ are known, $u$ is given via a representation formula (see, e.g., [10]).

Under some assumptions on $\Omega$ (cf. $\S 5$ ), the single-layer potential $V: H^{-1 / 2}(\Gamma) \rightarrow$ $H^{1 / 2}(\Gamma)$ is linear, bounded, symmetric and positive definite. $\left(H^{1 / 2}(\Gamma)\right.$ is the trace space of $H^{1}(\Omega)$ and $H^{-1 / 2}(\Gamma)=H^{1 / 2}(\Gamma)^{*}$, see $\S 5$ for details.) For $f \in H^{1}(\Gamma)$, Lax-Milgram's lemma guarantees that (1.1) has a unique solution $\phi \in H^{-1 / 2}(\Gamma)$, which then belongs to $L^{2}(\Gamma)[9]$. Moreover, by Cea's lemma, Galerkin methods like (Galerkin) BEM are quasi-optimally convergent. The simplest of such discretization schemes is described by a partition (a so-called mesh) $\pi=\left\{\Gamma_{1}, \ldots, \Gamma_{n}\right\}$ of the polygon $\Gamma$ in intervals (so-called elements) $\Gamma_{1}, \ldots, \Gamma_{n}$. Then

$$
S_{\pi}^{0}(\Gamma):=\left\{v_{h} \in L^{\infty}(\Gamma):\left.v_{h}\right|_{\Gamma_{j}} \in \mathbb{R} \quad \text { for all } j=1, \ldots, N\right\}
$$

denotes the linear space of piecewise constant functions and $h(\pi) \in S_{\pi}^{0}(\Gamma)$ is defined as the local mesh size, i.e., $\left.h(\pi)\right|_{\Gamma_{j}}:=\left|\Gamma_{j}\right|:=$ length of $\Gamma_{j}$.

Lax-Milgram's lemma guarantees a unique solution $\phi_{h} \in S_{\pi}^{0}(\Gamma)$ of the Galerkin equations, which are equivalent to

$$
R_{h}:=f-V \phi_{h} \perp S_{\pi}^{0}(\Gamma) .
$$

Here, $\perp$ means orthogonality in $L^{2}(\Gamma)$, i.e., $\int_{\Gamma} R_{h} \psi_{h} d s=0$ for all $\psi_{h} \in S_{\pi}^{0}(\Gamma)$. Note that $R_{h} \in H^{1}(\Gamma)$, so that the derivative $\frac{\partial}{\partial s} R_{h}=R_{h}^{\prime}$ of $R_{h}$ along $\Gamma$ with respect to the arclength exists and belongs to $L^{2}(\Gamma)$.

Theorem 1 ([4]). There exists a constant $c>0$ (depending only on $\Gamma$ ) such that for any $s \in[0,1]$ there holds

$$
\left\|\phi-\phi_{h}\right\|_{H^{-s}(\Gamma)} \leq c \cdot\left\|R_{h}^{\prime}\right\|_{L^{2}(\Gamma)}^{1-s}\left\|h(\pi) \cdot R_{h}^{\prime}\right\|_{L^{2}(\Gamma)}^{s} .
$$

An immediate consequence of Theorem 1 is

$$
\left\|\phi-\phi_{h}\right\|_{H^{-s}(\Gamma)} \leq c \cdot \max h(\pi)^{s} \cdot\left\|R_{h}^{\prime}\right\|_{L^{2}(\Gamma)}
$$

with $\max h(\pi):=\|h(\pi)\|_{L^{\infty}(\Gamma)}$. Compared with (1.5), the estimate (1.6) is reasonable if $\max h(\pi) / \min h(\pi)$ is not too large, $\min h(\pi):=\min \{h(\pi)(x): x \in \Gamma\}$. That means that there is a global constant $c_{u}$ such that for all meshes $\pi$ under consideration,

$$
\max h(\pi) / \min h(\pi)=\max _{j \neq k}\left|\Gamma_{j}\right| /\left|\Gamma_{k}\right| \leq c_{u} .
$$

We prove in this paper the reverse inequality of (1.6) and hence affirm the sharpness of the estimate in Theorem 1 for quasi-uniform meshes. 
Theorem 2. If $f$ is continuous and smooth on each side of $\Gamma$, there exist constants $c_{0}, h_{0}>0$ (depending only on $\Gamma, f$ and $c_{u}$ ) such that for all partitions $\pi$ of $\Gamma$ with $\max h(\pi)<h_{0}$ and $(1.7)$, and for $s \in[0,1]$, one has

$$
\max h(\pi)^{s} \cdot\left\|R_{h}^{\prime}\right\|_{L^{2}(\Gamma)} \leq c_{0} \cdot\left\|\phi-\phi_{h}\right\|_{H^{-s}(\Gamma)} .
$$

The rest of this paper is organized as follows: The general framework of a posteriori estimates presented in [4] is recalled and enlarged to cover an abstract complementary estimate in $\S 2$. The three ingredients of this estimate are (i) approximation properties (upper and lower bounds for the the best approximation), (ii) inverse assumptions and (iii) stability of the discretization of the trial functions in a certain sense. A more transparent implication is derived in $\S 3$ while the proof is given in $\S 4$. The abstract results are studied for Symm's integral equation in $\S 5$ and Theorem 2 is proved there. Two other applications concerning the hypersingular integral equation and an integral equation for some transmission problem are described in $\S \S 6$ and 7 . The paper concludes with miscellaneous comments in $\S 8$, including a comparison of the estimates in [4] and [5].

\section{Abstract Setting}

We recall the general approach of [4] for a posteriori error estimates, enlarge it and add a complementary abstract estimate.

Let $X_{1} \subset X_{0}$ and $Y_{1} \subset Y_{0}$ be real Banach spaces, and let $X_{\theta}:=\left[X_{0}, X_{1}\right]_{\theta}$ and $Y_{\theta}:=\left[Y_{0}, Y_{1}\right]_{\theta}$ be defined by interpolation of these spaces, $0 \leq \theta \leq 1$, cf. [2]. We briefly list a few properties inherited from interpolation, which are assumed in the sequel: There are positive constants $c_{\theta, X}$ and $c_{\theta, Y}$ such that for all $x \in X_{1}$ and $y \in Y_{1}$

$$
\|x\|_{X_{\theta}} \leq c_{\theta, X} \cdot\|x\|_{X_{0}}^{1-\theta} \cdot\|x\|_{X_{1}}^{\theta} \quad \text { and } \quad\|y\|_{Y_{\theta}} \leq c_{\theta, Y} \cdot\|y\|_{Y_{0}}^{1-\theta} \cdot\|y\|_{Y_{1}}^{\theta} .
$$

Let $L(X ; Y)$ denote the Banach space of linear bounded mappings between the Banach spaces $X$ and $Y$, and let $\|\cdot\|_{L(X ; Y)}$ be the corresponding operator norm. Then, for each $A_{j} \in L\left(X_{j} ; Y_{j}\right), j=0,1$, with $\left.A_{0}\right|_{X_{1}}=A_{1}$, the restriction $A_{\theta}=$ $A_{0} \mid X_{\theta}$ belongs to $L\left(X_{\theta} ; Y_{\theta}\right)$ and

$$
\left\|A_{\theta}\right\|_{L\left(X_{\theta} ; Y_{\theta}\right)} \leq\left\|A_{\theta}\right\|_{L\left(X_{0} ; Y_{0}\right)}^{1-\theta} \cdot\left\|A_{\theta}\right\|_{L\left(X_{1} ; Y_{1}\right)}^{\theta} .
$$

Let $A=A_{\theta}$ be such a mapping and assume, in addition, that $A_{\theta}: X_{\theta} \rightarrow Y_{\theta}$ is surjective and injective. Then, fix a right-hand side $f \in Y_{1}$ and the solution $u \in X_{\theta}$ of

$$
A u=f .
$$

We apply the Galerkin method to approximate $u$. Let $S_{h} \subset X_{1}$ and $T_{h} \subset Y_{0}^{*}$ be finite-dimensional subspaces such that there exists some $u_{h} \in S_{h}$ satisfying

$$
t_{h}\left(A u_{h}\right)=t_{h}(f) \text { for all } t_{h} \in T_{h} .
$$

The residual $R_{h}:=f-A u_{h}$ and the error $e_{h}:=u-u_{h}$ are related by the following estimate.

Theorem 3 ([4]). There exists $\rho \in Y_{0}^{*}$ satisfying

$$
\|\rho\|_{Y_{0}^{*}}^{2}=\left\|R_{h}\right\|_{Y_{0}}^{2}=\rho\left(R_{h}\right)
$$


and there holds

$$
\left\|e_{h}\right\|_{X_{\theta}} \leq c_{\theta, Y} \cdot\left\|A^{-1}\right\|_{L\left(Y_{\theta} ; X_{\theta}\right)} \cdot\left\|R_{h}\right\|_{Y_{1}}^{\theta} \cdot \inf _{t_{h} \in T_{h}}\left\|\rho-t_{h}\right\|_{Y_{0}^{*}}^{1-\theta} .
$$

Proof. To make the presentation self-contained, we give a brief outline of the proof. The existence of the (weighted) peak functional $\rho \in Y_{0}^{*}$ satisfying (2.5) follows from a well-known corollary of Hahn-Banach's theorem. Since $A=A_{\theta}: X_{\theta} \rightarrow Y_{\theta}$ is bijective, we have $e_{h}=A^{-1} R_{h}$ and

$$
\left\|e_{h}\right\|_{X_{\theta}} \leq\left\|A^{-1}\right\|_{L\left(Y_{\theta} ; X_{\theta}\right)} \cdot\left\|R_{h}\right\|_{Y_{\theta}} .
$$

By interpolation, $\left\|R_{h}\right\|_{Y_{\theta}} \leq c_{\theta, Y} \cdot\left\|R_{h}\right\|_{Y_{0}}^{1-\theta} \cdot\left\|R_{h}\right\|_{Y_{1}}^{\theta}$. The proof is concluded by standard duality arguments, using (2.4) and (2.5),

$$
\left\|R_{h}\right\|_{Y_{0}}^{2}=\rho\left(R_{h}\right)=\left(\rho-t_{h}\right)\left(R_{h}\right) \leq\left\|R_{h}\right\|_{Y_{0}} \cdot\left\|\rho-t_{h}\right\|_{Y_{0}^{*}}\left(t_{h} \in T_{h}\right) .
$$

This abstract estimate can be made precise, e.g., Theorem 3 implies Theorem 1 for Symm's integral equation (cf. $\S 5$ ). Since other examples are also covered by the given framework, we state the efficiency result in a related abstract form.

The following concepts (i)-(iii) are essential where we consider a family of Galerkin methods described by a family of discrete subspaces $\left(S_{h}: h \in I\right)$ and $\left(T_{h}: h \in I\right)$ of $X_{1}$ and $Y_{0}^{*}$, respectively.

(i) Approximation property. Assume that the solution $u \in X_{\theta}$ of (2.3) also belongs to $X_{1}$. Then, for each $h \in I$, let

$$
E\left(u, S_{h}\right):=\inf \left\{\left\|u-v_{h}\right\|_{X_{1}}: v_{h} \in S_{h}\right\}=\left\|u-\Pi_{h} u\right\|_{X_{1}}
$$

be the best approximation error in the norm of $X_{1}$ and let $\Pi_{h}: X_{1} \rightarrow S_{h}$ denote a projection such that $\Pi_{h} u$ is the best approximate in $S_{h}$. Assume $E\left(u, S_{h}\right)>$ 0 (provided the Galerkin method converges quasi-optimally, otherwise we obtain $u_{h}=u$ and this is the exceptional case we are not interested in) and define

$$
F\left(u, S_{h}\right):=\frac{\left\|u-\Pi_{h} u\right\|_{X_{0}}}{\left\|u-\Pi_{h} u\right\|_{X_{1}}}
$$

(ii) Inverse assumption. For each $h \in I$ let

$$
G\left(S_{h}\right):=\sup \left\{\frac{\left\|v_{h}\right\|_{X_{1}}}{\left\|v_{h}\right\|_{X_{0}}}: v_{h} \in S_{h} \backslash\{0\}\right\} .
$$

(iii) Stability. For each $h \in I$ let

$$
P_{h}: X_{0} \rightarrow S_{h} \text { be a projection }
$$

such that $P_{h} \in L\left(X_{0}, X_{0}\right)$ and $\left.P_{h}\right|_{X_{1}} \in L\left(X_{1}, X_{1}\right)$ with norms

$$
\left\|P_{h}\right\|_{j}:=\sup \left\{\frac{\left\|P_{h} v\right\|_{X_{j}}}{\|v\|_{X_{j}}}: v \in X_{j} \backslash\{0\}\right\}, \quad j=0,1 .
$$

Then, for $h, H \in I$, define

$$
\begin{aligned}
\delta\left(u, S_{h}, S_{H}\right):= & \frac{E\left(u, S_{H}\right)}{E\left(u, S_{h}\right)} \\
& \cdot\left(1+c_{\theta, X} \cdot\left\|P_{H}\right\|_{1}^{\theta} \cdot\left[F\left(u, S_{H}\right) G\left(S_{H}\right)\left\|P_{H}\right\|_{0}\right]^{1-\theta}\right) .
\end{aligned}
$$


Remark 1. For Symm's equation we have $X_{0}=H^{-1}(\Gamma), X_{1}=L^{2}(\Gamma)=Y_{0}, Y_{1}=$ $H^{1}(\Gamma)$, and, as we shall see below, by standard arguments, $E\left(u, S_{h}\right) \simeq F\left(u, S_{h}\right) \simeq$ $h, G\left(S_{h}\right) \simeq h^{-1}$. The projection $P_{h}$ will satisfy $\left\|P_{h}\right\|_{0},\left\|P_{h}\right\|_{1} \leq C$. In this case, if $H=\epsilon h$,

$$
\delta\left(u, S_{h}, S_{H}\right) \simeq C \epsilon,
$$

which is smaller than 1 for sufficiently small $\epsilon$.

Theorem 4. Let $A \in L\left(X_{1}, Y_{1}\right), h, H \in I$ with $S_{h} \subseteq S_{H}$; consider $u, u_{h} \in X_{1}$, $e_{h}:=u-u_{h}, R_{h}:=A e_{h} \in Y_{1}$, and assume (i)-(iii). If $\delta\left(u, S_{h}, S_{H}\right)<1$, then

$$
\left\|R_{h}\right\|_{Y_{1}} \leq \frac{\left\|P_{H}\right\|_{0}^{1-\theta} \cdot\left\|P_{H}\right\|_{1}^{\theta}}{1-\delta\left(u, S_{h}, S_{H}\right)} \cdot\|A\|_{L\left(X_{1} ; Y_{1}\right)} \cdot G\left(S_{H}\right)^{1-\theta} \cdot\left\|e_{h}\right\|_{X_{\theta}} .
$$

The proof of Theorem 4 is given in $\S 4$.

Remark 2. The complementary character of the two complementary inequalities of Theorems 3 and 4 should be emphasized: No assumption on the test functions or the residual (neither (2.4) nor bijectivity of $A: X_{\theta} \rightarrow Y_{\theta}$ ) is required in Theorem 4 , which are essential in Theorem 3. Conversely, the approximation, inverse, and stability properties of the trial spaces, the main ingredients in Theorem 4, play no role in Theorem 3 .

\section{Specialized Setting}

Since $E\left(u, S_{h}\right), F\left(u, S_{h}\right)$ and $G\left(S_{h}\right)$ can be bounded if $S_{h}$ are spline function spaces, we state in this section a more transparent form of the abstract estimate of Theorem 4. Let $\left(S_{h}: h \in I\right)$ be a family of subspaces of $X$, where the index $h$ is a positive parameter, say, $I \subset(0,1)$. Suppose $S_{h} \subset S_{H}$ for all $h, H \in I$ with $H<h$ and that $\bigcup_{h \in I} S_{h}$ is dense in $X_{1}$. Suppose that there exist positive constants $c_{\alpha}, c_{\beta}, c_{p}$ and real constants $\alpha, \beta$ such that for all $h \in I$

$$
\begin{aligned}
F\left(u, S_{h}\right) & \leq c_{\alpha} \cdot h^{\alpha}, \\
G\left(S_{h}\right) & \leq c_{\beta} \cdot h^{\beta}, \\
\left\|P_{h}\right\|_{0}^{1-\theta} \cdot\left\|P_{h}\right\|_{1}^{\theta} & \leq c_{p} .
\end{aligned}
$$

Corollary 1. Assume (3.1)-(3.3) and $\alpha+\beta \geq 0$. Define

$$
\begin{aligned}
& c_{1}:=2+2 c_{\theta, X} \cdot c_{p} \cdot c_{\alpha}^{1-\theta} \cdot c_{\beta}^{1-\theta}, \\
& c_{2}:=2 c_{p} \cdot\|A\|_{L\left(X_{1} ; Y_{1}\right)} \cdot c_{\beta}^{1-\theta} .
\end{aligned}
$$

Then, for each $h \in I$, we can find $H \in I$ with

$$
E\left(u, S_{H}\right) \leq \frac{1}{c_{1}} E\left(u, S_{h}\right) \quad \text { and } \quad H<h,
$$

and we have $\left\|R_{h}\right\|_{Y_{1}} \leq c_{2} \cdot H^{\beta(1-\theta)} \cdot\left\|e_{h}\right\|_{X_{\theta}}$.

Proof. Use the above notation in Theorem 2 to see that (3.4) implies $\delta\left(u, S_{h}, S_{H}\right) \leq$ $1 / 2$. Then, (2.8) proves the corollary.

As chosen in Corollary 1, $H$ depends highly on $h$ and we need more information on $E\left(u, S_{h}\right)$ to control this in (3.4). 
Corollary 2. In addition to the assumptions of Corollary 1 let there exist constants $\eta, q, h_{0}, 0<\eta, q<1$, such that for all $h \in I$ with $h<h_{0}$ we have

$$
E\left(u, S_{\eta \cdot h}\right) \leq q \cdot E\left(u, S_{h}\right) \quad \text { and } \quad \eta \cdot h \in I .
$$

Then, there exists $c_{0}>0$ such that for all $h \in I$ with $h<h_{0}$

$$
\left\|R_{h}\right\|_{Y_{1}} \leq c_{0} \cdot h^{\beta(1-\theta)} \cdot\left\|e_{h}\right\|_{X_{\theta}} \text {. }
$$

Proof. Use (3.5) and $H=\eta^{k} \cdot h$ in Corollary 1 with a natural number $k \geq$ $-\log \left(c_{1}\right) / \log (q)$.

Remark 3. The assumptions on $F\left(u, S_{h}\right), G\left(S_{h}\right)$ as well as an upper bound of $E\left(u, S_{h}\right)$, like $E\left(u, S_{h}\right) \leq c \cdot h^{\gamma}$, are standard tools in finite element and boundary element analysis (see, e.g., [19]). The assumption on the stability (concerning $c_{p}$ ) is sometimes used implicitly. The only additional assumption is some kind of lower bound of the best approximation error $E\left(u, S_{h}\right)$, e.g., $c^{\prime} \cdot h^{\gamma} \leq E\left(u, S_{h}\right)$, such that (3.5) holds: $E\left(u, S_{H}\right) \leq c \cdot H^{\gamma} \leq c \cdot \eta^{\gamma} h^{\gamma} \leq c \cdot \eta^{\gamma} / c^{\prime} E\left(u, S_{h}\right)$.

\section{Proof of Theorem 4}

We need two lemmas to convey the assumptions to the interpolation spaces.

Lemma 1. For any $v_{h} \in S_{h}, h \in I$, we have

$$
\left\|v_{h}\right\|_{X_{1}} \leq\left\|P_{h}\right\|_{0}^{1-\theta}\left\|P_{h}\right\|_{1}^{\theta} \cdot G\left(S_{h}\right)^{1-\theta} \cdot\left\|v_{h}\right\|_{X_{\theta}} .
$$

Proof. By interpolation of the projection $P_{h}$ as a mapping in $L\left(X_{j}, X_{1}\right)$ for $j=0,1$ we get as in (2.2) (where the image $X_{1}$ is fixed) that $P_{h} \in L\left(X_{\theta}, X_{1}\right)$ with

$$
\left\|P_{h}\right\|_{L\left(X_{\theta} ; X_{1}\right)} \leq\left\|P_{h}\right\|_{L\left(X_{0} ; X_{1}\right)}^{1-\theta} \cdot\left\|P_{h}\right\|_{1}^{\theta} .
$$

By the inverse assumption (ii), $\left\|P_{h}\right\|_{L\left(X_{0} ; X_{1}\right)} \leq\left\|P_{h}\right\|_{0} \cdot G\left(S_{h}\right)$. An application of the bound of $\left\|P_{h}\right\|_{L\left(X_{\theta} ; X_{1}\right)}$ (to $\left.v_{h}=P_{h} v_{h}\right)$ proves the lemma.

Lemma 2. For any $h \in I$ we have

$$
\left\|u-\Pi_{h} u\right\|_{X_{\theta}} \leq c_{\theta, X} \cdot F\left(u, S_{h}\right)^{1-\theta} \cdot\left\|u-\Pi_{h} u\right\|_{X_{1}} .
$$

Proof. Combine the approximation property with (2.1).

Proof of Theorem 4. Let $h, H \in I$ with $S_{h} \subset S_{H}$ and define $w_{H}:=\Pi_{H} u$. By continuity of $A=A_{1}: X_{1} \rightarrow Y_{1}$ we obtain

$$
\left\|R_{h}\right\|_{Y_{1}} \leq\|A\|_{L\left(X_{1} ; Y_{1}\right)} \cdot\left\|e_{h}\right\|_{X_{1}}
$$

and then focus on the error $e_{h}=u-u_{h}$. By the triangle inequality and Lemma 1 (with $v_{H}=w_{H}-u_{h} \in S_{H}$ ) we obtain

$$
\left\|e_{h}\right\|_{X_{1}} \leq E\left(u, S_{H}\right)+\left\|P_{H}\right\|_{0}^{1-\theta}\left\|P_{H}\right\|_{1}^{\theta} \cdot G\left(S_{H}\right)^{1-\theta} \cdot\left\|w_{H}-u_{h}\right\|_{X_{\theta}} .
$$

By the triangle inequality and Lemma 2 ,

$$
\left\|w_{H}-u_{h}\right\|_{X_{\theta}} \leq c_{\theta, X} \cdot F\left(u, S_{H}\right)^{1-\theta} \cdot E\left(u, S_{H}\right)+\left\|e_{h}\right\|_{X_{\theta}} .
$$

Combining (4.2), (4.3) and

$$
E\left(u, S_{H}\right) \leq \frac{E\left(u, S_{H}\right)}{E\left(u, S_{h}\right)} \cdot\left\|e_{h}\right\|_{X_{1}}
$$


one infers

$$
\begin{aligned}
& \left\|e_{h}\right\|_{X_{1}} \leq \delta\left(u, S_{h}, S_{H}\right) \cdot\left\|e_{h}\right\|_{X_{1}} \\
& \quad+\left\|P_{H}\right\|_{0}^{1-\theta} \cdot\left\|P_{H}\right\|_{1}^{\theta} \cdot G\left(S_{H}\right)^{1-\theta} \cdot\left\|e_{h}\right\|_{X_{\theta}} .
\end{aligned}
$$

Now, (2.8) follows from (4.1) and (4.4).

\section{Symm'S INTEGRAL EQUATION}

We use the notation from $\S 1$ and recall that $\Gamma$ is a polygon in $\mathbb{R}^{2}$. The Sobolev space $H^{s}\left(\mathbb{R}^{2}\right)$ is defined as usual [21] and

$$
\begin{aligned}
H^{s}(\Gamma) & :=\left\{\left.u\right|_{\Gamma} \in L^{2}(\Gamma): u \in H^{1 / 2+s}\left(\mathbb{R}^{2}\right)\right\}, \quad s>0 \\
H^{0}(\Gamma) & :=L^{2}(\Gamma), \\
H^{s}(\Gamma) & :=H^{-s}(\Gamma)^{*}, \quad s<0 \\
H_{0}^{s}(\Gamma) & :=\left\{u \in H^{s}(\Gamma): \int_{\Gamma} u d s=0\right\} \\
L_{0}^{2}(\Gamma) & :=H_{0}^{0}(\Gamma),
\end{aligned}
$$

where $H^{s}(\Gamma)^{*}$ is the dual of $H^{-s}(\Gamma)$ with respect to the extended inner product in $L^{2}(\Gamma)$. Further, $Y_{\theta}=H^{\theta}(\Gamma)$ is the interpolation space of $Y_{0}=L^{2}(\Gamma)$ and $Y_{1}=H^{1}(\Gamma)$ and $X_{\theta}=H^{\theta-1}(\Gamma)$ is the interpolation space of $X_{0}=H^{-1}(\Gamma)$ and $X_{1}=L^{2}(\Gamma), 0 \leq \theta \leq 1, c_{\theta, X}=c_{\theta, Y}=1$.

It is also known that $A:=V$ is a pseudodifferential operator and is a linear and bounded mapping between $H^{s-1}(\Gamma)$ and $H^{s}(\Gamma)$ for any $s \in[0,1]$ [9]. Throughout this paper, we assume $\operatorname{cap}(\Gamma)<1$, so that $V$ defines a positive definite bilinear form on $H^{-1 / 2}(\Gamma)^{2}[25,26,27]$. Here, $\operatorname{cap}(\Gamma)$ is the capacity (or conformal radius or transfinite diameter) of $\Gamma$ and $\operatorname{cap}(\Gamma)<1$ is satisfied if, for example, $\Omega$ is included in a disc with radius $<1$, which can be arranged by scaling. Moreover, by the regularity results in [9], $V: L^{2}(\Gamma) \rightarrow H^{1}(\Gamma)$ is bijective. Hence, $V$ satisfies the assumptions on $A$ in $\S 2$, and we obtain in particular Theorem 1.

Proof of Theorem 1. The assumptions of Theorem 3 are satisfied in the present case and $R_{h}=\rho$ is easily verified. Hence, with Theorem 3 and some standard estimation of the approximation error $R_{h}-\Pi_{h} R_{h}$, one proves (1.5) (cf. [4]).

In the next steps we prove the estimates described in $\S 3$, where it is sufficient that $f$ in (1.1) is continuous and $\left.f\right|_{\omega}$ belongs to $H^{3}(\omega)$ for each side $\omega$ of the polygon $\Gamma$. Then, $\phi=V^{-1}(f) \in L^{2}(\Gamma)$ and we are interested in its $L^{2}(\Gamma)$-best approximation error

$$
E\left(\phi, S_{h}\right)=\min \left\{\left\|\phi-\psi_{h}\right\|_{L^{2}(\Gamma)}: \psi_{h} \in S_{\pi}^{0}(\Gamma)\right\}=\left\|\phi-\Pi_{h} \phi\right\|_{L^{2}(\Gamma)} ;
$$

here, $\Pi_{h}$ is the orthogonal projection onto $S_{h}=S_{\pi}^{0}(\Gamma)$ in $L^{2}(\Gamma)$. If $\phi$ is constant, then $E\left(\phi, S_{h}\right)=0$ and $R_{h}=0$ indicates $\phi=\phi_{h}$. Thus, we may, and will, assume $\phi$ to be nonconstant in the following.

Proposition 1. Provided $\phi$ is not constant, there exist positive constants $\gamma, h_{0}$, $c_{\gamma}$, and $c_{\gamma}^{\prime}$ (depending only on $\Gamma, f$ and $c_{u}$ in (1.7)) such that $0<\gamma \leq 1$ and either

$$
c_{\gamma}^{\prime} \leq \max h(\pi)^{-\gamma} \cdot E\left(\phi, S_{h}\right) \leq c_{\gamma},
$$


or $\gamma=1 / 2$ and

$$
c_{\gamma}^{\prime} \leq-\max h(\pi)^{-1 / 2} \cdot \log ^{-1 / 2}(\max h(\pi)) \cdot E\left(\phi, S_{h}\right) \leq c_{\gamma},
$$

holds for all meshes $\pi$ with $\max h(\pi)<h_{0}$ and satisfying (1.7).

Proof. As it is proved in [10], the solution $\phi$ of (1.1) has the form

$$
\phi(x)=\phi_{0}(x)+\sum_{j=1}^{m} c_{j} \cdot \phi_{j}(x), \quad x \in \Gamma,
$$

where $\phi_{0} \in H^{2}(\Gamma)$ and the real constants $c_{j}$ depend on $f$, but the singular functions $\phi_{j}$ depend on the corners of the polygon only. Here, $\phi_{j}$ is of the form

$$
\phi_{j}(x)=r^{\beta_{j}} \cdot \chi_{j}(x) \quad \text { or } \quad \phi_{j}(x)=r^{\beta_{j}} \cdot \log (r) \cdot \chi_{j}(x),
$$

where $r$ is the Euclidean distance between $x$ and the nearest corner of $\Gamma$. Furthermore, $\chi_{j}$ is a smooth cutoff function with a small compact support which is one on a neighborhood of some corner of $\Gamma$. The exponent $\beta_{j}$ is determined by the interior angle $\omega_{j}$ at the related corner: $\beta_{j}+1=k_{j} \pi / \omega_{j}$ for some nonnegative integer $k_{j}$. Since $0<\omega_{j}<2 \pi, \omega_{j} \neq \pi$, we conclude $\beta_{j}>-1 / 2$. Moreover, the second case $\phi_{j}(x)=r^{\beta_{j}} \cdot \log r$ appears if $\beta_{j}+1=k_{j} \pi / \omega_{j}$ is integer, whence only if $\beta_{j} \geq 1$.

We split the proof into several steps considering $\phi(x)=r^{\beta}, \phi(x)=r^{\beta} \log r$, $\phi(x) \in H^{2}(\Gamma)$ first before we come back to the general case. We analyze the approximation properties locally, so $\phi$ is approximated on the real unit interval by $S_{h}^{0}(0,1)$ on a quasi-uniform mesh on $(0,1)$ described by a partition $0=x_{0}<x_{1}<$ $x_{2}<\cdots<x_{n}<x_{n+1}=1$. Define $m_{j}=h_{j}^{-1} \cdot \int_{x_{j}}^{x_{j+1}} \phi(x) d x=\left.\Pi_{h} \phi\right|_{\left(x_{j}, x_{j+1}\right)}$ and $h_{j}:=x_{j+1}-x_{j}$ for $j=0, \ldots, n$.

Case 1. Let $\phi(x)=x^{\beta}$ for $x \in(0,1)$, and $-1 / 2<\beta<1 / 2, \beta \neq 0$. Let $0 \leq a<a+h \leq 1$,Summary: " and consider the error $\|\phi-m\|_{L^{2}(a, a+h)}$, where $\phi$ is approximated by the constant $m=h^{-1} \cdot \int_{a}^{a+h} \phi(x) d x$. Some calculations show

$$
\begin{aligned}
\|\phi-m\|_{L^{2}(a, a+h)}^{2} & =a^{2 \beta+1} \cdot \eta(h / a) \quad(a>0), \\
\|\phi-m\|_{L^{2}(0, h)}^{2} & =\frac{\beta^{2}}{(1+\beta)^{2}(2 \beta+1)} \cdot h^{2 \beta+1} \quad(a=0),
\end{aligned}
$$

where

$$
\eta(\delta):=\frac{(1+\delta)^{2 \beta+1}-1}{2 \beta+1}-\frac{\left[(1+\delta)^{\beta}-1\right]^{2}}{\delta(1+\beta)^{2}} \quad(\delta>0) .
$$

A power series expansion of $\eta$ in $\delta$ shows

$$
\eta(\delta)=c_{1} \cdot \delta^{3}+\text { higher-order terms in } \delta
$$

with a positive constant $c_{1}$ (depending only on $\beta>-1 / 2$ ). Note that, the first exponent in (5.7) is expected to be three (because of linear convergence when a constant approximates a smooth function). Moreover, one can conclude from (5.7) and (5.5) that

$$
c_{2} \leq \eta(\delta) \cdot \delta^{-3} \leq c_{3} \quad \text { for all } \delta \in\left(0, c_{u}\right]
$$

with constants $c_{j}$ depending on $\beta$ and $c_{u}$. 
According to (5.5) and (5.6),

$$
\begin{aligned}
\left\|\phi-\Pi_{h} \phi\right\|_{L^{2}(\Gamma)}^{2}=h_{0}^{2 \beta+1} \cdot\left(\frac{\beta^{2}}{(1+\beta)^{2}(2 \beta+1)}\right. & \\
& \left.+\sum_{j=1}^{n}\left(x_{j} / h_{0}\right)^{2 \beta+1} \cdot \eta\left(h_{j} / x_{j}\right)\right) .
\end{aligned}
$$

Since $2-2 \beta>1$, we have with Riemann's Zeta function $\zeta$

$$
\sum_{j=1}^{n} j^{2 \beta-2} \leq \zeta(2-2 \beta)=\sum_{j=1}^{\infty} j^{2 \beta-2} .
$$

Using this, (5.8), (5.9), and (1.7), one finally obtains (5.2) for $\phi(x)=x^{\beta}$ and $S_{h}=S_{h}^{0}(0,1), \gamma=\beta+1 / 2$.

Case 2. Let $\phi(x)=x^{\beta}$ for $x \in(0,1)$ as before but now $\beta=1 / 2$. The above calculations remain true for $\beta=1 / 2$, in particular (5.8) and (5.9) hold, but the zeta function has a pole at 1 . Using the well-known formula

$$
\sum_{j=1}^{n} j^{-1}=\log n+C+O(1 / n) \quad(n \rightarrow \infty)
$$

involving Euler's constant $C=0.577 \ldots$ instead, the above arguments prove (5.3) for $\phi(x)=x^{1 / 2}$ and $S_{h}=S_{h}^{0}(0,1)$.

Case 3. Let $\phi \in H^{1}(0,1)$, e.g., $\phi(x)=x^{\beta} \log x$ for $\beta \geq 1$. Then, the estimate $\max h(\pi)^{-1} \cdot\left\|\phi-\Pi_{h} \phi\right\|_{L^{2}(\Gamma)} \leq c_{4}$ is well known. Conversely, let $\phi \in H^{2}(\omega)$ for at least a compact subinterval $\omega$ of one side of $\Gamma$ where $\phi$ is not constant. Then we have a compact nonempty subinterval $[a, b]$ where $\phi^{\prime} \in C([a, b])$ attains values between $c_{5}$ and $c_{6}$ with $c_{5} \cdot c_{6}>0$. As it is easily seen,

$$
c_{7} \cdot \min h(\pi) \leq\left\|\phi-\Pi_{h} \phi\right\|_{L^{2}([a, b])} \leq\left\|\phi-\Pi_{h} \phi\right\|_{L^{2}(\Gamma)}
$$

holds in this case for some positive constant $c_{7}$. This proves (5.2) for $\phi=\phi_{0}$, for $\phi(x)=x \cdot \log x$, and also for $\phi(x)=x^{\beta}$ with $\beta>1 / 2, S_{h}=S_{h}^{0}(0,1)$.

So far we proved for each summand in (5.4) an inequality

$$
c_{j}^{\prime} \leq-\max h(\pi)^{-\beta_{j}} \cdot \log (\max h(\pi))^{-\beta_{j}^{\prime}} \cdot\left\|\phi_{j}-\Pi_{h} \phi_{j}\right\|_{L^{2}(\Gamma)} \leq c_{j}
$$

with positive $c_{j}, c_{j}^{\prime}, \beta_{j}, \beta_{j}^{\prime}$ being independent of $h$.

The triangle inequality and (5.11) prove (5.2) or (5.3) for $\beta:=\min \left\{\beta_{0}, \ldots\right.$, $\left.\beta_{m}\right\}>0$. The proof of the upper bound is immediate while the proof of the lower bound uses that the terms with exponent $\beta$ are dominant and that $\beta=\beta_{j}$ is possible at most once at each corner. We omit the details.

Remark 4. Upper bounds for the best approximation error $E\left(\phi, S_{h}\right)$ are well known for spline functions. Indeed, regularity of $\phi$ can be measured in the convergence rate of $E\left(\phi, S_{h}\right)$ (see, e.g., [13, p. 358]):

$$
E\left(\phi, S_{h}\right)=O(\max h(\pi)) \quad \text { if and only if } \quad \phi \in H^{1}(\Gamma)
$$

provided we have a uniform mesh (i.e., $c_{u}=1$ ). In particular, this explains that (5.2) is false for $\phi(x)=x^{1 / 2}$. 
Remark 5. A model example is studied in [18] where, in particular, $E\left(\phi, S_{h}\right)$ is computed with $\phi(x)=x^{\beta}$ and $S_{h}$ consists of (possibly discontinues) piecewise polynomials of degree $p$ at most on a uniform mesh of $(0,1)$ with meshsize $h$. In particular it is proved (cf. arguments in [18, p. 603] and [18, Part 2, Corollary 2.1]) that the limits of

$$
h^{-p} \cdot E\left(\phi, S_{h}\right), \quad-h^{-p} \cdot \sqrt{\log (h)} \cdot E\left(\phi, S_{h}\right) \quad \text { and } \quad h^{-\beta-1 / 2} \cdot E\left(\phi, S_{h}\right)
$$

exist as positive real numbers as $h \rightarrow 0^{+}$for $p<\beta-1 / 2, p=\beta-1 / 2$ and $p>\beta-1 / 2$, respectively. This confirms Cases 1 and 2 in the proof of Proposition 1 for $p=0$ for a uniform mesh $\left(c_{u}=1\right)$ and extends the arguments to splines with polynomials of arbitrary degree.

Proposition 2. There holds $F\left(\phi, S_{h}\right) \leq \max h(\pi)$.

Proof. Note that $\Pi_{h}$ is the $L^{2}$-projection onto $S_{\pi}^{0}(\Gamma)$. Let $\sup _{\eta}$ denote the supremum among all nonzero $\eta$ in $H^{1}(\Gamma)$. Then we have

$$
\begin{aligned}
\left\|\phi-\Pi_{h} \phi\right\|_{H^{-1}(\Gamma)} & =\sup _{\eta} \frac{\int_{\Gamma}\left(\phi-\Pi_{h} \phi\right)\left(\eta-\pi_{h} \eta\right) d s}{\|\eta\|_{H^{1}(\Gamma)}} \\
& \leq \max h(\pi) \cdot\left\|\phi-\Pi_{h} \phi\right\|_{L^{2}(\Gamma)},
\end{aligned}
$$

using $\left\|\eta-\Pi_{h} \eta\right\|_{L^{2}(\Gamma)} \leq \max h(\pi) \cdot\left\|\eta^{\prime}\right\|_{L^{2}(\Gamma)}$ for all $\eta \in H^{1}(\Gamma)$.

Let prime or $\frac{\partial}{\partial s}$ denote the derivative along the straight sides of the polygon $\Gamma$ with respect to the arclength. Conversely, given $f \in L_{0}^{2}(\Gamma)$, let $I(f)$ be defined by integrating $f$ along $\Gamma$ with respect to the arclength. This defines $I(f)$ up to an additive constant on each side of $\Gamma$, which is chosen such that $I(f)$ is continuous at each corner. So far, $I(f) \in H^{1}(\Gamma)$ is defined up to an additive constant, which is fixed by $\int_{\Gamma} I(f) d s=0$. This defines $I: L_{0}^{2}(\Gamma) \rightarrow H_{0}^{1}(\Gamma)$.

Lemma 3 ([4, Lemma 3]). For all $s \in[0,1], I$ is an isomorphism between $H_{0}^{s-1}(\Gamma)$ and $H_{0}^{s}(\Gamma)$ with $\frac{\partial}{\partial s} I(f)=f$ for all $f \in H_{0}^{s-1}(\Gamma)$.

Besides $S_{\pi}^{0}(\Gamma)$, define

$$
S_{\pi}^{1}(\Gamma):=\left\{w_{h} \in H^{1}(\Gamma): w_{h}^{\prime} \in S_{\pi}^{0}(\Gamma)\right\},
$$

the linear space of continuous and piecewise linear functions with respect to a mesh $\pi$.

The following result shows $G\left(S_{h}\right) \leq C / h$.

Proposition 3. There exists a constant $c>0$ such that for all meshes $\pi$ with $\max h(\pi)<1$

$$
\begin{aligned}
\left\|w_{h}\right\|_{H^{1}(\Gamma)} & \leq c \cdot \min h(\pi)^{-1} \cdot\left\|w_{h}\right\|_{L^{2}(\Gamma)} \quad \text { for all } \quad w_{h} \in S_{\pi}^{1}(\Gamma), \\
\left\|\psi_{h}\right\|_{L^{2}(\Gamma)} & \leq c \cdot \min h(\pi)^{-1} \cdot\left\|\psi_{h}\right\|_{H^{-1}(\Gamma)} \quad \text { for all } \quad \psi_{h} \in S_{\pi}^{0}(\Gamma)
\end{aligned}
$$

Proof. The inverse inequality (5.13) is well known and easily proved by direct calculations on each element. According to Lemma $3, I: S_{\pi}^{0}(\Gamma) \cap L_{0}^{2}(\Gamma) \rightarrow S_{\pi}^{1}(\Gamma) \cap L_{0}^{2}(\Gamma)$ is an isomorphism, and (5.14) follows essentially from (5.13).

For each $\psi \in H^{-1}(\Gamma)$ define $P_{h} \psi \in S_{\pi}^{0}(\Gamma)$ by

$$
P_{h} \psi:=\psi^{0}+\frac{\partial}{\partial s} \Pi_{h}^{1} I\left(\psi-\psi^{0}\right) \quad \text { for } \quad \psi^{0}:=|\Gamma|^{-1} \int_{\Gamma} \psi d s
$$

where $\Pi_{h}^{1}$ is the $L^{2}$-projection onto $S_{\pi}^{1}(\Gamma)$ (see [6] for a similar construction). 
Proposition 4. The operator $P_{h}$ is a projection onto $S_{\pi}^{0}(\Gamma)$ which is bounded as a mapping between $H^{-1}(\Gamma)$ and $H^{-1}(\Gamma)$ or between $L^{2}(\Gamma)$ and $L^{2}(\Gamma)$.

Proof. It is known that $\Pi_{h}^{1}$ is continuous as a mapping between $L^{2}(\Gamma)$ and $L^{2}(\Gamma)$ and between $H^{1}(\Gamma)$ and $H^{1}(\Gamma)$ [12]. According to this and Lemma $3, P_{h}$ is linear and bounded as a mapping between $H^{-1}(\Gamma)$ and $H^{-1}(\Gamma)$ and between $L^{2}(\Gamma)$ and $L^{2}(\Gamma)$. Finally, direct calculations show $P_{h} \psi_{h}=\psi_{h}$ for each $\psi_{h} \in S_{\pi}^{0}$, i.e., $P_{h}$ is a projection onto $S_{\pi}^{0}(\Gamma)$.

Proof of Theorem 2. Using the above notation and that of $\S 3$ the Propositions 2-4 show (3.1)-(3.3) where, as usual, the index parameter $h$ is identified with max $h(\pi)$ for a mesh $\pi$ satisfying (1.7).

Given a mesh $\pi$, let $\eta=1 / k$ for an integer $k$ and define a new mesh by dividing each element $\Gamma_{j}$ of $\pi$ in $k$ pieces of length $\left|\Gamma_{j}\right| / k$. Then, the new mesh satisfies (1.7) as $\pi$ does. Moreover, according to Proposition 1, we obtain (3.5) with some $q$ which depends on $k, c_{\gamma}, c_{\gamma}^{\prime}$, and $\gamma$. A simple calculation, indicated in Remark 3, shows that we obtain $0<q<1$ by choosing $k$ large enough. Note that $\eta$ depends only on $c_{\gamma}, c_{\gamma}^{\prime}$ and $\gamma$. Therefore, Corollary 2 leads to Theorem 2 .

Remark 6. Note that a solution $u$ of (1.3) satisfies $\phi=\frac{\partial u}{\partial n} \in H_{0}^{-1 / 2}(\Gamma)$. Hence, we might be interested in determining $\phi \in H_{0}^{-1 / 2}(\Gamma)$ with

$$
\int_{\Gamma}(V \phi-f) \psi d s=0 \quad \text { for all } \quad \psi \in H_{0}^{-1 / 2}(\Gamma) .
$$

The corresponding numerical method is: Find $\phi_{h} \in S_{h}:=S_{\pi}^{0}(\Gamma) \cap L_{0}^{2}(\Gamma)$ with

$$
\int_{\Gamma}\left(V \phi_{h}-f\right) \psi_{h} d s=0 \quad \text { for all } \quad \psi_{h} \in S_{h}
$$

Equations (5.15) and (5.16) have unique solutions according to Lax-Milgram's lemma. In this case, one also obtains Theorems 1 and 2 from Theorems 3 and 4 .

\section{Hypersingular integral EQUATION}

The Neumann problem for the Laplacian

$$
\Delta u=0 \quad \text { in } \Omega \quad, \quad \frac{\partial u}{\partial n}=t_{0} \quad \text { on } \Gamma,
$$

is related to the hypersingular integral equation

$$
W v(x)=f(x) \quad(x \in \Gamma) .
$$

Here, $f$ can be computed from the Neumann data $t_{0}$ and $v=\left.u\right|_{\Gamma}$ is the trace of $u$. The hypersingular operator

$$
W v(x):=-\frac{1}{\pi} \frac{\partial}{\partial n_{x}} \int_{\Gamma} v(y) \frac{\partial}{\partial n_{y}} \log |x-y| d s_{y}
$$

is linear, bounded, symmetric and a Fredholm operator of index zero [9]. Since $W=$ $-\frac{\partial}{\partial s} V \frac{\partial}{\partial s}[22], W$ is positive definite between $H_{0}^{1 / 2}(\Gamma)$ and $H_{0}^{-1 / 2}(\Gamma)$. Furthermore, $A=W$ is a continuous mapping between $X_{1}=H_{0}^{1}(\Gamma)$ and $Y_{1}=L_{0}^{2}(\Gamma)$ as well as between $X_{0}=L_{0}^{2}(\Gamma)$ and $Y_{0}=H_{0}^{-1}(\Gamma)$. Let $L_{0}^{2}(\Gamma)$ be endowed with the usual norm in $L^{2}(\Gamma)$, but endow $H_{0}^{1}(\Gamma)$ with the equivalent $H^{1}(\Gamma)$-seminorm. 
Fix $f \in L_{0}^{2}(\Gamma)$ and the solution $v \in H_{0}^{1}(\Gamma)$ of $(6.1)$. Let $T_{h}=S_{h}=S_{\pi}^{1}(\Gamma) \cap H_{0}^{1}(\Gamma)$ as defined in (5.12) for a partition $\pi$ of the polygon $\Gamma$ with (1.7). Let $v_{h} \in S_{h}$ denote the Galerkin solution, i.e.,

$$
R_{h}:=f-W v_{h} \perp S_{\pi}^{1}(\Gamma),
$$

where $\perp$ means orthogonality in $L^{2}(\Gamma)$.

Theorem 5 ([4]). There exists a constant $c>0$ (depending only on $\Gamma$ ) such that for any $s \in[0,1]$ there holds

$$
\left\|v-v_{h}\right\|_{H^{s}(\Gamma)} \leq c \cdot\left\|R_{h}\right\|_{L^{2}(\Gamma)}^{s}\left\|h \cdot R_{h}\right\|_{L^{2}(\Gamma)}^{1-s} .
$$

For quasi-uniform meshes, $(6.3)$ is

$$
\left\|v-v_{h}\right\|_{H^{s}(\Gamma)} \leq c \cdot \max h(\pi)^{1-s}\left\|R_{h}\right\|_{L^{2}(\Gamma)} .
$$

As Theorem 5 follows from Theorem 3, we infer its converse from Theorem 4 .

Theorem 6. Assume that $f$ is smooth on each side of the polygon. Then there exist constants $c_{0}, h_{0}>0$ (depending only on $\Gamma, f$ and $c_{u}$ ) such that for all partitions $\pi$ of $\Gamma$ with $\max h(\pi)<h_{0}$ and (1.7) and for $s \in[0,1]$, there holds

$$
\max h(\pi)^{1-s} \cdot\left\|R_{h}\right\|_{L^{2}(\Gamma)} \leq c_{0} \cdot\left\|v-v_{h}\right\|_{H^{s}(\Gamma)} .
$$

Proof. Let $\Pi_{h}^{j}$ denote the $L^{2}$-projection onto $S_{\pi}^{j}(\Gamma)$ for $j=0,1$. Since we endowed $H_{0}^{1}(\Gamma)$ with the norm

$$
\|w\|_{H_{0}^{1}(\Gamma)}=\left(\int_{\Gamma}\left|w^{\prime}(s)\right|^{2} d s\right)^{1 / 2} \quad\left(w \in H_{0}^{1}(\Gamma)\right),
$$

the relation $E\left(v, S_{h}\right)=\left\|v-w_{h}\right\|_{H_{0}^{1}(\Gamma)}$ is equivalent to $v^{\prime}-w_{h}^{\prime} \perp S_{\pi}^{0}(\Gamma)$ for each $w_{h} \in S_{h}^{1}(\Gamma)$. Thus $w_{h}^{\prime}=\Pi_{h}^{0} v^{\prime}$ and $E\left(v, S_{h}\right)=\left\|v^{\prime}-\Pi_{h}^{0} v^{\prime}\right\|_{L^{2}(\Gamma)}$. Since the derivative $v^{\prime}$ of the solution $u$ of the Neumann problem, $v=\left.u\right|_{\Gamma}$, has a similar decomposition as $\phi$ in (5.4), this and the results of $\S 5$ verify the assumptions of Corollary 2. We omit the details.

Remark 7. A second proof of Theorem 6 is possible using the close relation between $V$ and $W$ and a modification of Theorem 2. Indeed, $\phi:=v^{\prime}$ and $\phi_{h}:=v_{h}^{\prime}$ satisfy $V \phi=F:=I(f)$ and

$$
\int_{\Gamma} V\left(\phi_{h}\right) \psi_{h} d s=\int_{\Gamma} F \psi_{h} d s \text { for all } \psi_{h} \in S_{\pi}^{0}(\Gamma) \cap L_{0}^{2}(\Gamma) .
$$

Thus, $\phi \in L_{0}^{2}(\Gamma)$ and $\phi_{h} \in L_{0}^{2}(\Gamma)$ are solutions of problem (5.15) and (5.16), respectively. Then, arguing as in Remark 6 , we can obtain Theorem 6 from Theorem 2 .

\section{INTEGRAL EQUATION FOR A TRANSMISSION PROBLEM}

In the transmission problem we seek harmonic functions $u_{1}$ and $u_{2}$ in an interior and exterior domain $\Omega$ and $\mathbb{R}^{2} \backslash \Omega$, respectively, with a prescribed jump $(f, g)$ of their Cauchy data at the polygonal boundary $\Gamma=\partial \Omega$ (see, e.g., $[11,6]$ for details). This transmission problem is equivalently related to a boundary integral equation

$$
H\left(\begin{array}{c}
v \\
\phi
\end{array}\right)=\frac{1}{2}(1+H)\left(\begin{array}{c}
f \\
g
\end{array}\right)
$$


[11], where $(f, g) \in H^{1}(\Gamma) \times L^{2}(\Gamma)$ is given and

$$
H:=\left(\begin{array}{cc}
-K & V \\
W & K^{\prime}
\end{array}\right): H^{s}(\Gamma) \times H^{s-1}(\Gamma) \rightarrow H^{s}(\Gamma) \times H^{s-1}(\Gamma) .
$$

Here, $V$ and $W$ are given in (1.2) and (6.2), respectively, and the double-layer potential and its adjoint are defined by

$$
\begin{aligned}
K v(x) & :=-\frac{1}{\pi} \int_{\Gamma} v(y) \frac{\partial}{\partial n_{y}} \log |x-y| d s_{y}, \\
K^{\prime} \phi(x) & :=-\frac{1}{\pi} \int_{\Gamma} \phi(y) \frac{\partial}{\partial n_{x}} \log |x-y| d s_{y} .
\end{aligned}
$$

The operator $H$ is linear, bounded and bijective for $s \in[0,1]$ and (7.1) has a unique solution $(v, \phi) \in H^{1}(\Gamma) \times L^{2}(\Gamma)$ (see $\left.[11,6]\right)$. Define $S_{h}:=S_{\pi}^{1}(\Gamma) \times S_{\pi}^{0}(\Gamma)$ and $T_{h}:=S_{\pi}^{0}(\Gamma) \times S_{\pi}^{1}(\Gamma)$ for a partition $\pi$ of the polygon $\Gamma$. The Galerkin BEM for (7.1) reads: Find $\left(v_{h}, \phi_{h}\right) \in S_{h}$ such that the residual

$$
\left(R_{1}, R_{2}\right):=\frac{1}{2}(1+H)\left(\begin{array}{c}
f \\
g
\end{array}\right)-H\left(\begin{array}{c}
v_{h} \\
\phi_{h}
\end{array}\right) \in H^{1}(\Gamma) \times L^{2}(\Gamma)
$$

satisfies $0=\int_{\Gamma}\left(R_{1} \cdot \psi_{h}+R_{2} \cdot w_{h}\right) d s$ for all $\left(\psi_{h}, w_{h}\right) \in T_{h}$.

Theorem 7 ([4]). There exists a constant $C>0$ such that, for $0 \leq s \leq 1$,

$$
\left\|\left(\begin{array}{c}
v \\
\phi
\end{array}\right)-\left(\begin{array}{c}
v_{h} \\
\phi_{h}
\end{array}\right)\right\|_{H^{s}(\Gamma) \times H^{s-1}(\Gamma)} \leq C \cdot\left\|\left(\begin{array}{c}
R_{1}^{\prime} \\
R_{2}
\end{array}\right)\right\|_{L^{2}(\Gamma)^{2}}^{1-s} \cdot\left\|h(\pi) \cdot\left(\begin{array}{c}
R_{1}^{\prime} \\
R_{2}
\end{array}\right)\right\|_{L^{2}(\Gamma)^{2}}^{s} .
$$

While Theorem 7 is a consequence of Theorem 3, its converse estimate is concluded from the complementary results presented in $\S \S 2$ and 3 .

Theorem 8. If $f$ and $g$ are smooth, there exist constants $c_{0}, h_{0}>0$ (depending only on $\Gamma, f, g$ and $\left.c_{u}\right)$ such that for all partitions $\pi$ of $\Gamma$ with $\max h(\pi)<h_{0}$ and (1.7), and for $s \in[0,1]$, there holds

$$
\max h(\pi)^{s}\left\|\left(\begin{array}{l}
R_{1}^{\prime} \\
R_{2}
\end{array}\right)\right\|_{L^{2}(\Gamma)^{2}} \leq c_{0} \cdot\left\|\left(\begin{array}{c}
v \\
\phi
\end{array}\right)-\left(\begin{array}{c}
v_{h} \\
\phi_{h}
\end{array}\right)\right\|_{H^{s}(\Gamma) \times H^{s-1}(\Gamma)} .
$$

Proof. It can be checked that the assumptions of Corollary 2 are satisfied as in the previous cases. One combines arguments of $\S \S 5$ and 6 for $\phi$ and $v$ and uses regularity results from [11]. We omit the details.

\section{Comments}

8.1. Efficiency. Once the Galerkin solution $\phi_{h} \in S_{\pi}^{0}(\Gamma)$ is known, compute

$$
a_{j}^{2}=\int_{\Gamma_{j}}\left[\left(V \phi_{h}\right)^{\prime}(x)-f^{\prime}(x)\right]^{2} d s_{x}
$$

where, e.g., $V \phi_{h}(x)$ is calculated analytically and the integral along $\Gamma_{j}$ is approximated numerically; $h_{j}=\left|\Gamma_{j}\right|$. For $s=1 / 2$, the upper bound in Theorem 1 is $C \cdot B_{1}$, where $C$ is a constant and

$$
B_{1}:=\left(a^{2} \cdot \sum_{j=1}^{n} a_{j}^{2} \cdot h_{j}^{2}\right)^{1 / 4}, \quad a^{2}:=\sum_{j=1}^{n} a_{j}^{2} .
$$

Since $\int_{\Gamma} V(\cdot)(\cdot) d s$ defines an inner product which induces an equivalent norm in $H^{-1 / 2}(\Gamma)$, the quantity

$$
\gamma_{h}:=B_{1}^{-1} \cdot\left(\int_{\Gamma} V\left(\phi-\phi_{h}\right)\left(\phi-\phi_{h}\right) d s\right)^{1 / 2}
$$


is called efficiency quotient. We say that an error estimate is efficient if its efficiency quotient $\gamma_{h}$ satisfies $0<c_{0} \leq \gamma_{h} \leq c_{1}<\infty$ for all discretizations under consideration. Using this concept we proved in this paper that the a posteriori estimates obtained in [4] are efficient for BEM on quasi-uniform meshes (in two dimensions) and gave a general guideline in $\S \S 2$ and 3 on how to analyze this in other examples.

8.2. Numerical tests. The efficiency quotients $\gamma_{h}$ are computed for various numerical examples reported in $[5,6,7,8]$. From this, the efficiency results of this paper are confirmed; one observes efficiency in practice.

8.3. Comparison with another estimate. Another a posteriori error estimate is proved in [5] which bounds the error (in the $H^{-1 / 2}(\Gamma)$-norm) from above by $C \cdot B_{2}$, where $C$ is a constant and

$$
B_{2}:=\sum_{j=1}^{n} h_{j}^{1 / 2} \cdot a_{j}
$$

(notation as in §8.1). Numerical computations reported in [5] show a slow decrease of the related efficiency quotient as $n$ tends to infinity, even on quasi-uniform meshes. This "loss of efficiency" is indicated by the following example. Imagine that the residual is uniformly distributed on a uniform mesh $\pi$, i.e., suppose $a_{1}=$ $\cdots=a_{n}$ and $h_{1}=\cdots=h_{n}$. Then, $B_{1} \cdot \sqrt{n}=B_{2}$. According to the efficiency of $B_{1}$, this shows that $B_{2}$ overestimates the error by a factor $\sqrt{n}$, whence $B_{2}$ is not efficient in this model situation. However, if singularities occur, the estimate in [5] could be efficient.

8.4. Restrictions on the mesh. The condition of a quasi-uniform discretization (1.7) is used several times in the proofs. For an arbitrary mesh we have (1.6) and, according to (2.8) and (5.13),

$$
\min h(\pi)^{s} \cdot\left\|R^{\prime}\right\|_{L^{2}(\Gamma)} \leq c_{0} \cdot\left\|\phi-\phi_{h}\right\|_{H^{-s}(\Gamma)}
$$

in Theorem 2 (assuming that $\delta$ is small). Hence, the methods of this paper used for the proof of efficiency give the impression that one needs (1.7). One way of overcoming this difficulty is to take local properties of the operator $A$ into account. These appear to be unavailable in the literature for the operators studied here on polygons.

8.5. Further applications. The results in $\S \S 5-7$ are stated for the simplest discretization only. It is known that Propositions 2-4 also hold for spline functions of higher degree, and this is expected for an (adapted version of) Proposition 1 as well (cf. Remark 5). Indeed, the essential additional condition is (3.5), which may be proven in many other situations where $u$ is not an arbitrary function in $X_{1}$ but, e.g., is related to a solution of a partial differential equation and then inherits much more regularity. Therefore, it is expected that (3.5) is true for a larger class of discretizations, including problems in three dimensions, and is not restricted to solutions which are related to the Laplace equation.

\section{REFERENCES}

1. I. Babuška and A. Miller, A feedback finite element method with a posteriori error estimation, Comput. Methods Appl. Mech. Engrg. 61 (1987), 1-40. MR 88d:73036

2. J. Bergh and J. Löfström, Interpolation spaces, Springer, Berlin, 1976. MR 58:2349 
3. C. Carstensen, Adaptive boundary element methods and adaptive finite element and boundary element coupling, Boundary Value Problems and Integral Equations in Nonsmooth Domains (M. Costabel, M. Dauge, S. Nicaise, eds.), Proc. Conf. at the CIRM, Luminy, Lecture Notes in Pure and Appl. Math. vol. 167, Marcel Dekker, 1995, pp. 47-58. CMP 95:03

4. C. Carstensen and E. P. Stephan, A posteriori error estimates for boundary element methods, Math. Comp. 64 (1995), 483-500. MR 95f:65211

5. __ Adaptive boundary element methods for some first kind integral equations, SIAM J. Numer. Anal. (accepted for publication 1994).

6. _ Adaptive boundary element methods for transmission problems, J. Austral. Math. Soc. Ser. B (accepted for publication 1995).

7. _ Adaptive coupling of boundary elements and finite elements, Math. Modelling Numer. Anal. (accepted for publication 1995).

8. C. Carstensen, S. Funken, and E. P. Stephan, On the adaptive coupling of FEM and BEM in 2-d-elasticity, Preprint, Institut für Angewandte Mathematik, Universität Hannover, 1993.

9. M. Costabel, Boundary integral operators on Lipschitz domains: Elementary results, SIAM J. Math. Anal. 19 (1988), 613-626. MR 89h:35090

10. M. Costabel and E. P. Stephan, Boundary integral equations for mixed boundary value problems in polygonal domains and Galerkin approximation, Banach Center Publ. 15 (1985), 175-251. MR 88f:35037

11. , A direct boundary integral equation method for transmission problems, J. Math. Anal. Appl. 106 (1985), 367-413. MR 86f:76045

12. M. Crouzeix and V. Thomée, The stability in $L_{p}$ and $W_{p}^{1}$ of the $L_{2}$-projection onto finite element function spaces, Math. Comp. 48 (1987), 521-532. MR 88f:41016

13. R. A. DeVore and G. G. Lorenz, Constructive approximation, Springer, Berlin, 1993. MR 95f: 41001

14. K. Eriksson and C. Johnson, An adaptive finite element method for linear elliptic problems, Math. Comp. 50 (1988), 361-383. MR 89c:65119

15. K. Eriksson and C. Johnson, Adaptive finite element methods for parabolic problems I. A linear model problem, SIAM J. Numer. Anal. 28 (1991), 43-77. MR 91m:65274

16. B. Faehrmann, Lokale a-posteriori-Fehlerschätzer bei der Diskretisierung von Randintegralgleichungen, Ph.D. thesis, University of Kiel, FRG, 1993.

17. N. Heuer, $h p$-Versionen der Randelementemethode, Ph.D. thesis, University of Hannover, FRG, 1992.

18. W. Gui and I. Babuška, The $h, p$ and $h-p$ versions of the finite element method in 1 dimension, Numer. Math. 49 (1986), Part I, 577-612, Part II, 613-657, Part III, 659-683. MR 88b:65130a; MR 88b:65130b; MR 88b:65130c

19. G. C. Hsiao and W. L. Wendland, The Aubin-Nitsche lemma for integral equations, J. Integral Equations 3 (1981), 299-315. MR 83j:45019

20. C. Johnson and P. Hansbo, Adaptive finite element methods in computational mechanics, Comput. Methods Appl. Mech. Engrg. 101 (1992), 143-181. MR 93m:65157

21. J. L. Lions and E. Magenes, Non-homogeneous boundary value problems and applications, Vol. I, Springer, Berlin-Heidelberg-New York, 1972. MR 50:2670

22. J. C. Nedelec, La méthode des élements finis appliquée aux equations intégrales de la physique, First meeting AFCET-SMF on applied mathematics Palaiseau, Vol. 1, 1978, pp. 181-190.

23. E. Rank, Adaptive boundary element methods, Boundary Elements 9, Vol. 1 (C. A. Brebbia, W. L. Wendland and G. Kuhn, eds.), Springer-Verlag, Heidelberg, 1987, pp. 259-278. CMP 21:03

24. J. Saranen and W. L. Wendland, Local residual-type error estimates for adaptive boundary element methods on closed curves, Appl. Anal. 48 (1993), 37-50. MR 95e:65112

25. I. H. Sloan and A. Spence, The Galerkin method for integral equations of the first kind with logarithmic kernel: theory, IMA J. Numer. Anal. 8 (1988), 105-122. MR 90d:65230a

26. E. P. Stephan and W. L. Wendland, Remarks to Galerkin and least squares methods with finite elements for general elliptic problems, Manuscripta geodaetica 1 (1976), 93-123. MR 58:25012

27. E.P. Stephan, W.L. Wendland and G.C. Hsiao, On the integral equation method for the plane mixed boundary value problem of the Laplacian, Math. Methods Appl. Sci. 1 (1979), 265-321. MR 82e:31003 
28. R. Verfürth, A review of a posteriori error estimation and adaptive mesh-refinement techniques, Preprint, 1993.

29. W.L. Wendland and De-hao Yu, Adaptive boundary element methods for strongly elliptic integral equations, Numer. Math. 53 (1988), 539-558. MR 89h:65194

30. _ A posteriori local error estimates of boundary element methods with some pseudodifferential equations on closed curves, J. Comput. Math. 10 (1992), 273-289. MR 93d:65105

Fachbereich Mathematik, Technische Hochschule Darmstadt, D 64283 Darmstadt, GERMANY

E-mail address: carstensen@mathematik.th-darmstadt.de 